\title{
Huangjing (Polygonati rhizoma) is an emerging crop with great potential to fight chronic and hidden hunger
}

\author{
Donghong Chen, Zhigang Han \& Jinping Si \\ State Key Laboratory of Subtropical Silviculture, Zhejiang A\&F University, Hangzhou 311300, China
}

Received May 27, 2021; accepted June 2, 2021; published online June 17, 2021

Citation: Chen, D., Han, Z., and Si, J. (2021). Huangjing (Polygonati rhizoma) is an emerging crop with great potential to fight chronic and hidden hunger. Sci
China Life Sci 64, 1564-1566. https://doi.org/10.1007/s11427-021-1958-2

According to an annual flagship report produced jointly by the FAO and several other organizations, almost 690 million people, or 8.9 percent of the world's population, were still suffering from chronic hunger in 2019 (FAO et al., 2020). Moreover, the disastrous COVID-19 pandemic has severely threatened food security, and this may add between 83 and 132 million people to the total global number of undernourished in 2020. On the other hand, more than two billion people suffer from hidden hunger; this is usually associated with growth and developmental problems, mental impairment, poor overall health, and chronic diseases (Siddique et al., 2021). According to the IDF Diabetes Atlas (9th edition, 2019), there are 463 million diabetic patents in the world, with China ranking first in terms of the number of patients, at approximately 116.4 million. The global number is projected to reach an estimated 578 million by 2030 and 700 million by 2045 (www.diabetesatlas.org). Therefore, the goal of food production has been transformed from "eating fully" to "eating healthily", and hidden hunger has become a new hot topic that has received widespread attention from the international scientific community (Gashu et al., 2021; Jiang et al., 2021; Siddique et al., 2021).

Major crop domestication began approximately 12,000 years ago (Siddique et al., 2021). FAO (2019) revealed that just nine (sugar cane, maize, rice, wheat, potatoes, soybeans, oilpalm fruit, sugar beet, and cassava) of 6,000 plant species that have been cultivated for food account for $66 \%$ of the

*Corresponding author (email: 1ssjp@163.com) total crop production by weight. Three major cereals (maize, wheat, and rice) supply $60 \%$ of the global food energy intake. Siddique et al. (2021) reported that a rich variety of nutritious "forgotten crops" in Asia constitute a diverse cropping portfolio, which offers a viable and promising approach to close the current gaps in production and nutrition. Intriguingly, Si and Zhu (2021) found that Huangjing (Polygonati rhizoma) is emerging as a new generation crop that offers high yield and nutrition but does not require fertile land for growth, thus offering great potential to combat chronic and hidden hunger.

Huangjing was first recorded in the Chinese pharmaceutical monograph Sheng Nong's Herbal Classic, and its name derives from "its absorption of the essence from the earth". Huangjing is a traditional and classic dual-purpose plant for both food and medicine, and it has a good taste and can be consumed in large quantities over a long time. It has been cultivated and has served as a food substitute for more than 2,000 years in China. Given current cultivation technology, standard planting in the forest can produce $1,500 \mathrm{~kg}$ dry product per hectare sustainably (Figure 1).

Modern research has shown that Huangjing rhizome does not contain starch and is rich in non-starch polysaccharides, oligosaccharides, steroids, triterpenoid saponins, flavonoids, and other nutritional and functional components ( $\mathrm{Si}$ and $\mathrm{Zhu}$, 2021). It has the effects of tonifying Qi and nourishing Yin, invigorating the spleen, moistening the lungs, and benefiting the kidneys. In particular, non-starch polysaccharides and fructooligosaccharides contained in Huangjing rhizome have 

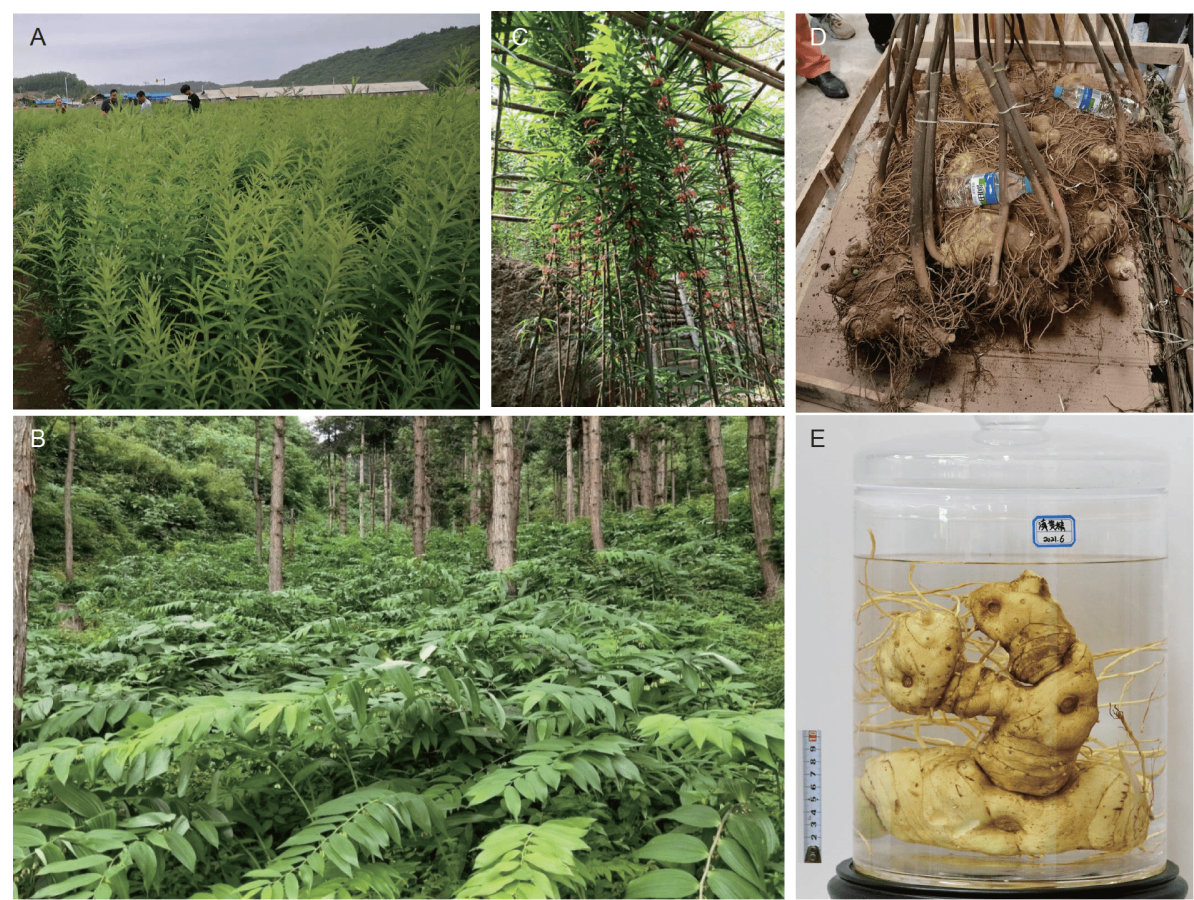

Figure 1 (Color online) The cultivation of Huangjing (Polygonati rhizoma). A, Polygonatum sibiricum planting in mountain regions of Liaoning Province. B, P. cyrtonema planting in forests of Hunan Province. C, P. kingianum planting in gardens of Yunnan Province. D, A representative 8-year-old P. kingianum plant with an $86 \mathrm{~kg}$ rhizome. E, Partial samples from (D).

the same effect as resistant starch in reducing postprandial blood glucose. Moreover, the rhizome can regulate blood glucose and enhance immunity by promoting insulin secretion and improving insulin sensitivity. It is one of the most cost-effective food therapy products for solving the problem of hidden hunger, particularly for patients with diabetes.

According to the Pharmacopoeia of the People's Republic of China, Huangjing consists of three basic species, Polygonatum sibiricum, $P$. kingianum, and $P$. cyrtonema (Chinese Pharmacopoeia Commission, 2020), which almost covers the areas suitable for artificial planting in China. It is suitable for large-scale in-forest cultivation in subtropical, temperate and cold temperate zones. Huangjing is a perennial plant that does not need to be sown every year, it has a long growing season, and it displays broad stress resistance. It can be planted once and harvested continuously, in line with the modern breeding concept of establishing a diversified perennial grain-cropping system that helps realize the development of sustainable agriculture (Tian et al., 2021). The natural polyploidy and aneuploidy of Huangjing provide natural materials for ploidy breeding. In-forest planting eliminates the need for good arable farmland, competition for forest land, and warehouse storage of Huangjing production. "Food storage in the forest" further strengthens a "food crop production strategy based on farmland management and technological application" and provides Chinese wisdom to ensure world food security and the transformation and upgrading of the food industry.
Huangjing is not only important for eradicating both chronic and hidden hunger, it also provides a novel insight into future genetic improvement of other crops through dissecting specific functional genes and pathways based on new technologies including genomics, phenomics, synthetic biology, gene editing, and plant transformation. Avenues for exploration include (i) elucidating the mechanism of starch deficiency and non-starch polysaccharide biosynthesis in Huangjing's rhizome and laying the foundation for the biofortification of other food crops, such as fructan-rich wheat/ sweet corn; (ii) dissecting the mechanism of shade photosynthetic efficiency of high-yield Huangjing to generate the possibility of transforming major crops such as potato/sweet potato to grow in the forest and thereby increase land use efficiency; and (iii) Clarifying the mechanism underlying that Huangjing is perennial and its rhizome is not rotten, and providing a reference for a perennial breeding strategy for existing annual crops, such as potato/sweet potato.

Compliance and ethics The author(s) declare that they have no conflict of interest.

Acknowledgements This work was supported by the Major Science and Technology Projects of Zhejiang Province (2021C02020).

\section{References}

Chinese Pharmacopoeia Commission. (2020). Pharmacopoeia of the People's Republic of China (Part I) (in Chinese). 2020 Ed. Beijing: China Pharmaceutical Science and Technology Press. 
FAO. (2019). The State of the World's Biodiversity for Food and Agriculture. In: Bélanger, J., and Pilling, D., eds. FAO Commission on Genetic Resources for Food and Agriculture Assessments. Rome.

FAO, IFAD, UNICEF, WFP, and WHO. (2020). The State of Food Security and Nutrition in the World 2020. In: Transforming Food Systems for Affordable Healthy Diets. Rome.

Gashu, D., Nalivata, P.C., Amede, T., Ander, E.L., Bailey, E.H., Botoman, L., Chagumaira, C., Gameda, S., Haefele, S.M., Hailu, K., et al. (2021). The nutritional quality of cereals varies geospatially in Ethiopia and Malawi. Nature 594, 71-76.

Jiang, L., Strobbe, S., Van Der Straeten, D., and Zhang, C. (2021).
Regulation of plant vitamin metabolism: backbone of biofortification for the alleviation of hidden hunger. Mol Plant 14, 40-60.

Si, J.P., and Zhu, Y.X. (2021). Polygonati rhizoma - a new high-quality crop with great potential and not occupying farmland (in Chinese). Sci Sin Vitae 51.

Siddique, K.H.M., Li, X., and Gruber, K. (2021). Rediscovering Asia's forgotten crops to fight chronic and hidden hunger. Nat Plants 7, 116122.

Tian, Z., Wang, J.W., Li, J., and Han, B. (2021). Designing future crops: challenges and strategies for sustainable agriculture. Plant J 105, 11651178 . 